\title{
Impact of Finger Position on Pinch Strength
}

\author{
Walukonis $\mathrm{KR}^{1 *}$, Beasley $\mathrm{J}^{2}$, Powers $\mathrm{J}^{3}$, Boerema \\ $\mathbf{R}^{4}$ and Anderson $\mathrm{K}^{5}$ \\ ${ }^{1}$ Special Education Department, Traverse Bay Area \\ Intermediate School District, USA \\ ${ }^{2}$ Department of Occupational Therapy, Grand Valley State \\ University, USA \\ ${ }^{3}$ Special Education Department, Wexford-Missaukee \\ Intermediate School District, USA \\ ${ }^{4}$ Rehabilitation Department, Cottage Rehabilitation \\ Hospital, USA \\ ${ }^{5}$ Department of Statistics, Grand Valley State University, \\ USA \\ *Corresponding author: Walukonis KR, Special \\ Education Department, Traverse Bay Intermediate School \\ District, 1101 Red Dr., Traverse City, MI 49684, USA
}

Received: January 18, 2017; Accepted: February 10,

2017; Published: February 13, 2017

\section{Abbreviations}

B\&L: Bernadette and Linda; ASHT: The American Society of Hand Therapists; IP: Interphalangeal; SPSS: Statistical Package for the Social Sciences

\section{Introduction}

Pinch strength measurement is a key point of interest to a myriad of health professionals working in hand therapy. A direct relationship between pinch strength and function has been demonstrated and illustrates the importance of hand strength in clinical practice [12]. Clinicians, especially those interested in preventing deformities and preserving functional performance, utilize pinch strength as an assessment tool and outcome measure. Specifically, pinch strength measurement using the "gold standard" Bernadette and Linda (B\&L) pinch gauge has been found to be psychometrically sound with established reliability and validity $[3,4]$. Clinicians and researchers utilize pinch strength to compare clients' strength with normative standards, characterize upper extremity impairment, document improvement in strength and overall function, determine goals, demonstrate industry research outcomes, and provide appropriate interventions [1-6].

Position of the upper extremity during hand strength measurement has been a topic of discussion for decades. In 1981, The American Society of Hand Therapists' (ASHT) suggested norms for arm position during grip strength tests [7]. Fess and Moran recommended, "the patient should be seated with his shoulder adducted and neutrally rotated, the elbow flexed to $90^{\circ}$, and the forearm and wrist in neutral position" [7]. However, while these recommendations may be valuable, they do not necessarily provide a standard testing position specific to pinch strength. A measurement protocol specific to pinch strength was not included in the ASHT recommendations, nor has it been established in the literature thus far. Several studies have addressed the impact of certain factors on pinch strength as well as provided recommendations for standardizing pinch strength positioning independent of grip strength; however, positioning the finger on the bridge or the groove of the pinch gauge has not been carefully described. Therefore, additional normative studies specific to hand and forearm positioning are needed to establish a comprehensive standard measurement protocol for the assessment of pinch strength.

One two-part study addressed the effect of various upper extremities positioning on pinch strength with 20 healthy male students aged 20 to 34 years [8]. Results indicated no effect of shoulder position on pinch strength measurements, but a large effect of elbow position on pinch strength measurements when the elbow was flexed 120 degrees or more. Secondly, Halpern and Fernandez examined forearm and wrist positions [8]. This experiment included 27 combinations involving three different pinches (lateral, tip, and three-jaw-chuck), three forearm positions, and three wrist positions. Results of this study indicated that for all three types of pinch combined, maximum flexion and extension of the wrist resulted in decreased pinch strength measurements. The pronated forearm position also showed decreased pinch strength measurements (up to $7 \%$ ), as compared with supinated and neutral forearm positions.

Stegink Jansen and colleagues researched the effect of three forearm positions on lateral, tip, and three-jaw-chuck pinch measurements [9]. This study agreed with an earlier study by Woody and Mathiowetz, which found that forearm position, did not affect three-jaw-chuck pinch measurements [9-10]. However, unlike those researchers as well as Halpern and Fernandez, Stegink Jansen and her colleagues found differences in lateral and tip pinch measurements between the different forearm positions [8-10]. They found measurements of lateral pinch to be lowest in the supinated position, while tip pinch measurements were lowest when the forearm was pronated. Although these differences were noted, none of the differences were found to be statistically significant (largest effect size .144). The researchers used these findings to suggest that "standardization of forearm position is recommended when measuring pinch strength, but not required" [9].
Phys Med Rehabil Int - Volume 4 Issue 1 - 2017 ISSN : 2471-0377 | www.austinpublishing group.com Walukonis et al. @ All rights are reserved
Citation: Walukonis KR, Beasley J, Powers J, Boerema R and Anderson K. Impact of Finger Position on Pinch Strength. Phys Med Rehabil Int. 2017; 4(1): 1108. 


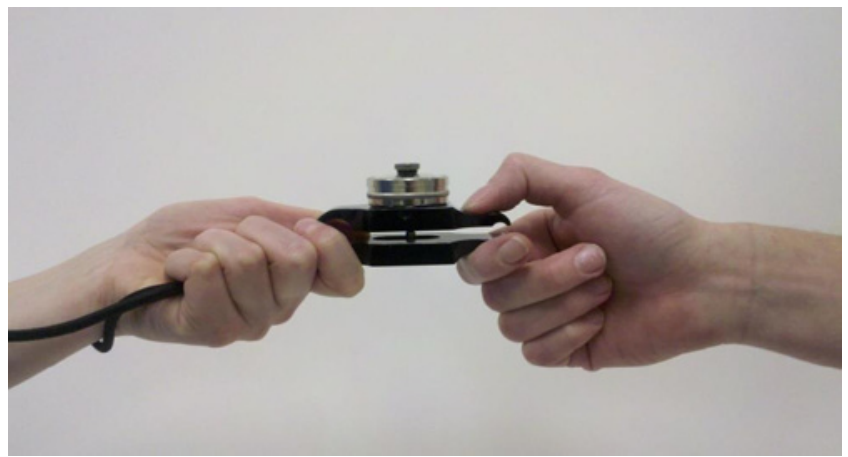

Figure 1: Lateral pinch on the groove of a B\&L Engineering pinch gauge.

They also suggested that the lack of standardization regarding finger placement on the bridge or groove of a B\&L pinch gauge, could be a confounding factor when interpreting results of current, recent, and future studies.

Although little research has been done on the effect of finger position on pinch strength measurements, two studies have addressed factors specific to the hand. In one study, McCoy and Dekerlegand addressed the lack of standardization for positioning of the ulnar three digits during tip pinch with 76 healthy volunteers [11]. They found

that pinch strengths were larger when the fingers were flexed, which agreed with findings by Hook and Stanley [12]. These researchers suggested that lack of standardization could greatly impact pinch strength measurements because the values are small, so even small differences could largely impact pinch measurement scores. In another study, Apfel sought to determine if thumb interphalangeal (IP) joint position, flexed or extended, effects lateral pinch measurements [13]. This study examined two IP joint positions, flexed or extended. Findings included significant differences in pinch strength measurements depending on IP joint position when all ASHT positioning standards were followed. For females and males, IP joint flexion showed an increase in strength by $28-30 \%$ and $36-38 \%$ respectively as compared to IP joint extension measurements.

Currently, no studies in the literature have focused how finger and thumb positioning on the pinch gauge impacts pinch strength. The potential problem may be due to how clinicians guide clients to place fingers and the thumb on the pinch gauge versus calibration standards of the pinch gauge. B\&L Engineering pinch gauges are calibrated with a digital force gauge by placing the pinch gauge into the force gauge at the groove. Therefore, the owner of B\&L, Lee Barnes, recommends that clients place fingers on the groove of the pinch gauge (Figure 1) when taking pinch measurements. Regardless of this recommendation, many clinicians feel that placing fingers and the thumb on the bridge of the gauge (Figure 2) is a more natural position. There were no studies found specifying the area of contact between the tip of the finger and thumb, but authors who presented illustrations of the test position showed that subjects pressed the bridge of the pinch meter rather than the groove [9]. The current study sought to determine if this discrepancy is clinically relevant and potentially introduce finger positioning recommendations.

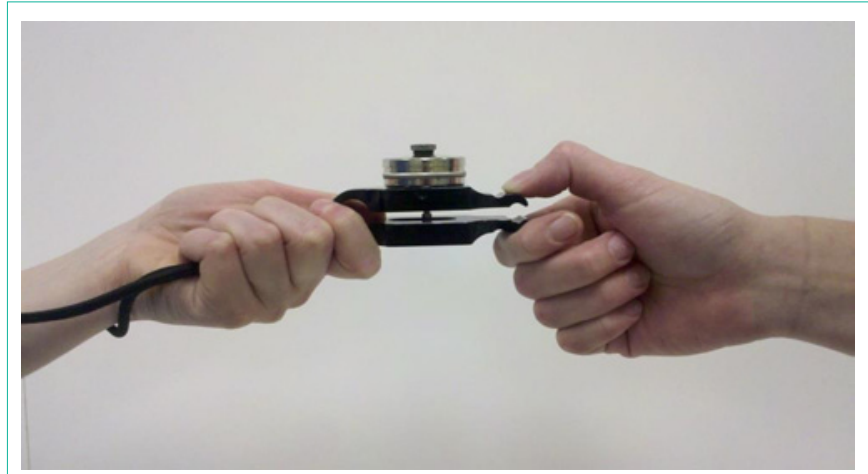

Figure 2: Lateral pinch on the bridge of a B\&L Engineering pinch gauge.

\section{Purpose of the Study}

The aim of the present study was to demonstrate whether finger position impacts three common pinch strengths using a B\&L Engineering pinch gauge. Further, it was intended that this research add to the existing literature of normative studies demonstrating the need for industry standardization of positioning with a pinch gauge during assessment. The hypothesis is that there is no significant difference in lateral, tip, and three-jaw-chuck pinch strength when measured with fingers placed on the bridge or groove of a B\&L Engineering pinch gauge.

\section{Materials and Methods}

\section{Study design}

This study utilized a crossover design with randomization of exposure to bridge versus groove positioning. The researchers sought to determine how this finger positioning on the pinch gauge affected the participants' tip, lateral, and three-jaw-chuck pinch strength.

\section{Participants}

A power analysis was conducted using $G^{\star}$ Power 3.1.9.2 based on use of a two-tailed paired t-test. To detect a moderate effect at a 5\% significance level with $80 \%$ power, it was determined that a sample size of at least 34 would be needed. Thirty-six participants were recruited to better correspond to the order randomization scheme described below in Procedures and Materials. Study participants were volunteers recruited from XXX University's (XXXX) student body, faculty, and guests using posters and faculty's verbal announcements. The study included 36 healthy adults (9 males, 27 females), aged 1949. Of these participants, 33 were right hand dominant and three were left hand dominant. Exclusion criteria included neurological or other dysfunction disorders of one or both upper extremities, history of upper extremity surgery or impairment within the last 12 months, and inability to follow English commands.

\section{Equipment}

The $0-30$ pound $B \& L$ Engineering pinch gauge with one pound increments was utilized in this study. This instrument was chosen for use in our study because it has high test-retest reliability, very high inter-rater reliability, demonstrated validity, and is known as the "gold standard" for pinch strength assessment" [7]. The one B\&L pinch gauge that was used in this study was newly purchased, calibrated by the manufacturer, and not used prior to data collection. 
Table 1: Mean pinch strength and p-values of statistical analysis.

\begin{tabular}{|c|c|c|c|c|}
\hline $\begin{array}{c}\text { Pinch } \\
\text { Type }\end{array}$ & $\begin{array}{c}\text { Bridge } \\
\text { (Mean } \pm \text { SD) }\end{array}$ & $\begin{array}{c}\text { Groove } \\
\text { (Mean } \pm \text { SD) }\end{array}$ & $\begin{array}{c}\text { Statistical Test } \\
\text { ( } \mathbf{p} \text {-value) }\end{array}$ & $\begin{array}{c}95 \% \\
\text { Confidence } \\
\text { Interval }\end{array}$ \\
\hline Lateral & $18.3 \pm 3.7$ & $18.6 \pm 3.8$ & $\begin{array}{c}\text { Paired T-test } \\
(p=0.510)\end{array}$ & $(-0.90,0.46)$ \\
\hline TJC & $16.6 \pm 3.5$ & $17.3 \pm 3.9$ & WSR test $(p=0.059)$ & $(-1.57,0.12)$ \\
\hline Tip & $11.2 \pm 2.7$ & $11.1 \pm 3.4$ & $\begin{array}{c}\text { Paired T-test } \\
(p=0.656)\end{array}$ & $(-0.59 .0 .92)$ \\
\hline
\end{tabular}

Measurements in pounds, TJC=Three-jaw-chuck, WSR=Wilcoxon Signed Ranks

\section{Procedures and materials}

This study was submitted to the XXXX Human Research Review Committee. After approval, this study was performed at the XXXX (XXX) in XX, X. On the day of testing, the researchers verbally explained the research procedures and an informational sheet detailing potential risks and benefits was given to each participant. The participants were blinded to the research hypothesis. The order of pinch was randomly assigned among participants with each order combination being represented equally. This was done to eliminate any potential order or fatigue effects. Prior to data collection, the researchers listed order combinations on demographic forms. Order combinations included pinch type (tip, lateral, three-jawchuck) and starting finger position (bridge or groove). Thus, there were 12 possible order combinations, and since 36 participants were partaking in the study, each pinch combination was written on 36 different demographic forms. After equally distributing orders onto the demographic forms, the forms were randomized so that on the day of testing, each participant was simply given the next demographic form in the pile. Each participant pinched the gauge a total of six times; once on the bridge and once on the groove for each type of pinch. Using one trial as the outcome score was selected to limit fatigue because research has shown no significant difference in pinch strength measurements when the mean of three trials, best of three trials, and one trial have been used to collect data $[3,9,14]$.

Patients were instructed to be seated with shoulder adducted and neutrally rotated, elbow flexed at $90^{\circ}$ and the forearm and wrist in neutral position, according to the guidelines by ASHT [7]. Literature also suggests no significant difference in pinch strength when the wrist is extended up to $30^{\circ}$ so slight variations in wrist position up to $30^{\circ}$ extension were permissible [15]. Additionally, the ulnar fingers and the IP joint of the thumb were flexed during the pinch measurement because research suggests these positions result in greater pinch force $[11,13]$. This testing position was visually estimated, and then maintained throughout the testing process with verbal feedback from the researchers. One trained rater performed the pinch strength testing. Each participant then was given one submaximal pinch warm-up in the first pinch position that was randomly assigned because this type of warm-up has been found to result in increased strength measurements [16]. Participants were given 15-second rest breaks between position changes, which was determined to be adequate by Trossman and Li (1989) [17]. Pinch measurements were taken with the dominant hand only because research has found a correlation between ability to complete activities of daily living and pinch strength for the dominant hand only [18]. The rater encouraged the participant to squeeze as hard as possible during each trial by saying "go, go, go, stop", as the use of consistent instructions is important for standardization of the test protocol [19]. This contraction time was no more than three seconds, which is supported by Smith and Lukens [20].

\section{Data analysis}

Pounds of force used during maximum pinch were gathered from the B\&L Engineering pinch gauge. Researchers entered data into Statistical Package for the Social Sciences (SPSS) version 20 and utilized the parametric paired t-test or the nonparametric Wilcoxon signed ranks test to analyze the data for each pinch type. The ShapiroWilk test of normality was used along with histograms and boxplots to determine the appropriate test. An alpha level of 0.05 was used to determine significance. Additionally, equivalence intervals were used to demonstrate a lack of substantial difference between measurements taken on the bridge or groove. A substantial difference was defined as one-unit (one pound) difference between measurements taken on the groove or bridge, giving an equivalence interval of $(-1,1)$. One pound was chosen because it is the smallest unit of measurement that can be read on the pinch gauge.

\section{Results and Discussion}

Table 1 depicts mean strengths and standard deviations for each type of pinch and finger position on the gauge as well as summarizes the results of statistical analysis.

\section{Lateral pinch}

Effect of position: There was no significant effect of finger position $(\mathrm{p}=.51)$.

Equivalence: The 95\% confidence interval for the difference in mean pinch strength between bridge and groove was $(-0.90,0.46)$; therefore, equivalence was found within one pound for lateral pinch.

\section{Three-Jaw-Chuck pinch}

Effect of position: There was no significant effect of finger position $(\mathrm{p}=.059)$. Three-jaw-chuck pinch strength was observed to be slightly higher on average with fingers on the groove.

Equivalence: The $95 \%$ confidence interval for the difference in mean pinch strength between bridge and groove was $(-1.57,0.12)$, which does not demonstrate equivalence for three-jaw-chuck pinch.

\section{Tip pinch}

Effect of position: There was no significant effect of finger position $(\mathrm{p}=.656)$.

Equivalence: The $95 \%$ confidence interval for the difference in mean pinch strength between bridge and groove was $(-0.59,0.92)$; therefore, equivalence was found within one pound for tip pinch.

\section{Other findings}

Neither hand dominance nor gender significantly affected tip, lateral or three-jaw-chuck pinch strengths.

\section{Discussion}

No statistically significant differences in mean pinch strength were found between bridge and groove digit placement for any of the three pinch types. Distinct from wrist position, forearm position, thumb interphalangeal position, and ulnar finger position, pinching on the bridge or groove may result in a similar tip pinch strength measurement on a B\&L engineering pinch gauge $[8,9,11,13]$. Additionally, equivalence was found within one pound for tip and lateral pinch, but not for three-jaw-chuck. 
The present study results are influential because they reveal that the discrepancy between calibration measurement and clinical measurement is not significant, implying that inter-rater reliability is not compromised. Clinicians may have increased confidence that measurements will be accurate regardless of whether they, or other healthcare personnel, guide clients to place fingers on the bridge or groove of the pinch gauge. Such information informs clinical practice by indicating that B\&L Engineering's recommendation to use a conversion factor of 0.9 may not be necessary [9]. Because finger position does not appear to impact pinch strength, perhaps the standard positioning on the bridge or groove could be determined by additional factors such as ease of positioning or patients' preference for comfort. For instance, positioning on the bridge is found to be easier to accomplish clinically as well as more comfortable per informal comments from this study's participants [9].

There were several limitations to this study. A small convenience sample of volunteers from XX compromises generalizability and statistical power of findings. The rater was not blinded to the research hypothesis, which could have consciously or unconsciously resulted in biased outcomes or reporting of outcomes. Although the researchers utilized the same new B\&L pinch gauge for the study, the researchers did not verify the calibration of this pinch gauge directly prior to data collection. According to Fess, this can result in more than acceptable measurement error and the researchers caution that results may not generalize to pinch gauges other than the B\&L [21]. Additionally, we chose to visually estimate upper extremity positioning during the procedure rather than to record goniometric measurements or provide stabilization.

Future research should be focused on conducting similar normative studies using a larger sample size and varied populations. Other pertinent populations include those with hand impairment or disability as well as older adults. These populations are relevant based on the findings in the literature which suggest that the relationship between hand strength and functional activities changes with these variables [22-25]. In future studies, a qualitative component involving the participants' perceptions of pinching on the bridge or groove may aid in understanding the effect of this variable on pinch strength measurements or lack thereof.

\section{Conclusions}

Although standardization of finger position is ideal and recommended, variations in finger position between the bridge and groove of the B\&L Engineering pinch gauge may result in similar pinch strength measurements. We do recommend a standardized test position be established and that all research and clinical documentation include a description of the testing position. Further research on this topic is needed to support the findings from this study, especially with regard to the impact of finger position on threejaw-chuck pinch strength since equivalence was not established.

\section{Acknowledgements}

The researchers would like to thank XXX, for overseeing the study at each phase and sharing her expertise regarding inter-rater reliability. Additionally, the researchers would like to extend a special thanks to XXX, for her assistance in creating an informational sheet for the participants of the study, and XXX, for providing us with valuable information concerning the calibration of the B\&L
Engineering pinch gauge.

\section{References}

1. Rice MS, Leonard C, Carter M. Grip strengths and required forces in accessing everyday containers in a normal population. Am J Occup Ther. 1998; 52: 621-626

2. Chang JH, Wu M, Lee CL, Guo YL, Chiu HY. Correlation of return to work outcomes and hand impairment measures among workers with traumatic hand injury. J Occup Rehabil. 2010; 21: 9-16.

3. MacDermid JC, Kramer JF, Woodbury MG, McFarlane RM, Roth JH. Interrater reliability of pinch and grip strength measurements in patients with cumulative trauma disorders. J Hand Ther. 1994; 7: 10-14.

4. Mathiowetz V, Vizenor L, Melander D. Comparison of baseline instruments to the jamar dynamometer and the B\&L engineering pinch gauge. Occup Ther J Res. 2000; 20: 147-161.

5. Mathiowetz V, Kashman N, Volland G, Weber K, Dowe M, Rogers S. Grip and pinch strength: Normative data for adults. Arch Phys Med Rehabil. 1985; 66: 69-74.

6. Ranganathan VK, Siemionow V, Sahgal V, Liu JZ, Yue GH. Skilled finger movement exercise improves hand function. J Gerontol. 2001; 56: M518-522.

7. Mathiowetz V, Weber K, Volland G, Kashman N. Reliability and validity of grip and pinch strength evaluations. J Hand Surg. 1984; 9: 222-226.

8. Halpern CA, Fernandez JE. The effect of wrist and arm postures on peak pinch strength. J Hum Ergol. 1996; 25: 115-130.

9. Stegink Jansen CW, Kocian Simper V, Stuart HG, Pinkerton HM. Measurement of maximum voluntary pinch strength: effects of forearm position and outcome score. J Hand Ther. 2003; 16: 326-336.

10. Woody R, Mathiowetz V. Effect of forearm position on pinch strength measurements. J Hand Ther. 1988; 1: 124-126.

11. McCoy W, Dekerlegand J. Effect of the position of ulnar three digits on thumb to index tip to tip pinch strength [Abstract]. J Hand Ther. 2011; 24: 379.

12. Hook WE, Stanley JK. Assessment of thumb to index pulp to pulp pinch grip strengths. J Hand Surg. 1986; 11: 91-92.

13. Apfel E. The effect of thumb interphalangeal joint position on strength of key pinch. J Hand Surg. 1986; 11: 47-51.

14. Hamilton A, Balnave R, Adams R. Grip strength testing reliability. J Hand Ther. 1994; $7:$ 163-170

15. Kraft GH, Detels PE. Position of function of the wrist. Arch Phys Med Rehabil. 1972; 53: 272-275

16. Marion R, Niebuhr BR. Effect of warm-up prior to maximal grip contractions. J Hand Ther. 1992; 7: 143-146.

17. Trossman PB, Li PW. The effect of duration of intertrial rest periods on isometric grip strength performance in young adults. Occup Ther J Res. 1989; 9: $362-378$

18. Rajan P, Premkumar R, Rajkumar $P$, Richard J. The impact of hand dominance and ulnar and median nerve impairment on strength and basic daily activities. J Hand Ther. 2005; 18: 40-45.

19. Richards L, Palmiter-Thomas P. Grip strength measurement: A critical review of tools, methods, and clinical utility. Crit Rev Phys Rehabil Med. 1996; 8: 87-109.

20. Smith DA, Lukens SA. Stress effects of isometric contraction in occupational therapy. Occup Ther J Res. 1983; 3: 222-242.

21. Fess EE. A method for checking jamar dynamometer calibration. J Hand Ther. 1987; 1: 28-32.

22. Puh U. Age-related and sex-related differences in hand and pinch grip strength in adults. Int J Rehabil Res. 2010; 33: 4-11.

23. Carmeli E, Patish H, Coleman R. The aging hand. J Gerontol. 2003; 5: 146152. 
24. Bagis S, Sahin G, Yapici Y, Cimen OB, Erdogan C. The effect of hand osteoarthritis on grip and pinch strength and hand function in postmenopausa women. Clin Rheumotol. 2003; 22: 420-424.
25. Guimaraes de Oliveira D, Aruin AS, Jose dos Santos M. Grip force control in individuals with hand osteoarthritis. J Hand Ther. 2011; 24: 345-354.
Phys Med Rehabil Int - Volume 4 Issue 1 - 2017

ISSN : 2471-0377 | www.austinpublishing group.com

Walukonis et al. (@) All rights are reserved
Citation: Walukonis KR, Beasley J, Powers J, Boerema R and Anderson K. Impact of Finger Position on Pinch Strength. Phys Med Rehabil Int. 2017; 4(1): 1108. 